\title{
Automated Nucleic Biosensors - A Key to High Resolution Monitoring of Marine Phytoplankton
}

\author{
Katja Metfies \\ Alfred Wegener Institute for Polar and Marine Research \\ Am Handelshafen 12 \\ 27570 Bremerhaven \\ Sonja Diercks,Friedhelm Schröder,Wilhelm Petersen \\ GKSS Research Center \\ Max Planck Strasse 1 \\ 21502 Geesthacht \\ Thomas Hanken \\ iSiTEC GmbH \\ Stresemann Strasse 46 \\ 27570 Bremerhaven
}

ABSTRACT

\begin{abstract}
Changes in plankton community structures in response to climate change and the climatic sensitivity of species are currently driving topics in marine research. In the marine environment phytoplankton consists of major primary producers, but also harmful algae that can negatively influence marine ecosystems. It is expected that climate related environmental change could result in changes in the abundance, spatial distribution, biogeography or dominance of phytoplankton species. In order to evaluate consequences of climate change for marine ecosystems it is necessary to possess high resolution information in time and space on current abundances and patterns within the phytoplankton. However, the generation of these data is constrained by a variety of reasons like the size or insufficient morphological markers of the taxa and the costs to provide samples with high spatiotemporal resolution.
\end{abstract}

In the past decade the application of biosensor technology has gained significant impact in respect to microbial analysis. More and more publications describe the development of molecular sensors dedicated to the detection of microbial organisms. In the EU FP6project ALGADEC a portable semi-automated biosensor-system has been developed in order to facilitate the detection of toxic algae in the field. This device enables the electrochemical detection of microalgae from watersamples in less than two hours, without the need of expensive equipment. This device is a prototype device that serves as a cornerstone of a new molecular based strategy for the monitoring of phytoplankton. Currently we are working on the adaptation of the biosensor to the surveillance of key species in the North Sea. And, we aim at a full automation of the system in order to provide an autonomous monitoring tool for phytoplankton. In the Future an autonomous biosensor can be combined with present in situ measurement systems for the marine environment like the FerryBox-system of the GKSS. This would serve the need for technologies that allow high resolution monitoring of marine phytoplankton in order to evaluate consequences of environmental change in the oceans.

\section{INTRODUCTION}

As fundamental components of the Earth systems, oceans and their inventories are important indicators of climate change. In fact, marine ecosystems are already affected by climate change [1;2] and it is expected that change within ecosystems (e.g. in food webs or community composition) will continue if prevailing predictions of further climate change like increased temperatures or ocean acidification are fulfilled. In general the knowledge on the consequences of the warming trend for marine food webs is only patchy. In particular this is true for the base of marine food webs that comprises planktonic organisms (size: $0.2 \mu \mathrm{m}-200 \mu \mathrm{m}$ ) like the phytoplankton or microzooplankton. However, they are fundamental for the functioning of marine ecosystems. This group of species comprises major primary producers of the ocean. Hence, it is expected that environmental change related reorganisation or shifts in the diversity and seasonality of species at the base of the planktonic food web would strongly impact the quantity and quality of food for higher trophic levels, e.g. via changes in the food spectrum. The fundamental ecological role of plankton (size fraction $<200 \mu \mathrm{m}$ ) for marine food webs makes it necessary to assess the consequences of climate change for 
this group of organisms. The necessity of marine plankton observation to assess consequences of climate change is challenged in various ways. These are difficulties to provide field samples with high spatiotemporal resolution and difficulties with the surveillance (species identification and enumeration) of species in the pico-, nano-, or microplankton fraction. As a consequence, especially the pico- and nanoplankton fraction has been understudied in the past. However, the introduction of molecular methods has facilitated the investigation of the biodiversity at the base of the marine food web, especially the assessment of taxa that are missing distinct morphological features. Many publications have shown the power of the analysis of ribosomal genes (rRNA-genes) to gain new insights into the phylogeny and for taxonomical identification of both prokaryotic and eukaryotic micro organisms [3]. The genes coding for the rRNA are particularly well suited for phylogenetic analysis and taxonomical identification, because they are universally present in all cellular organisms. Furthermore, rRNA genes are of relatively large size and contain both highly conserved and variable regions with no evidence for lateral gene transfer [4]. The analysis of the $18 \mathrm{~S}$ rDNA circumvents the selective step of laboratory cultivation [5]. The continually growing number of available algal 18S rDNA-sequences, e.g., in the Ribosomal Database Project (RDP) [6] and phylogenetic analysis make it possible to design hierarchical sets of probes that specifically target the 18S-rDNA from higher taxonomic levels down to the species level [7]. The probes can be used in combination with a wide variety of hybridization based methods, such as RNAbased nucleic acid biosensors or DNA-microarrays (Phylochips).

Nucleic acid biosensors and DNA-microarrays are chip based formats that allow a parallel identification and quantification of multiple taxa in a single experiment. The identification is based on solid phase hybridization of molecular probes, immobilized to the surface of the sensor chips to the rRNA or rDNA of the target species [8]. The application of molecular sensing technologies like nucleic acid biosensors or DNA-microarrays would greatly contribute to solve the problems connected to the observation of marine microbes. They constitute a tool box that allows quick, cost effective and species specific surveillance of plankton in time and space, which is indispensable for the generation of spatiotemporal high resolution observation data.

Despite the fundamental role of phytoplankton for marine ecosystems there is only a relatively small number of marine long time series worldwide. However, one major long term marine observation programme is operated by the Sir Alistair Hardy Foundation for Ocean Science in Plymouth, UK. The Continous Plankton Recorder (CPR) Survey's marine observation programme and its sister surveys provide largescale information on marine plankton distribution, mainly in the North Atlantic and the North Sea. Unfortunately, the CPRapproach is restricted to the survey of zooplankton and bigger phytoplankton e.g. diatoms. It omits ecological relevant phytoplankton groups e.g. the Cryptophyceae or Prasinophyceae, which belong to the pico- and nanoplankton fraction. In contrast, an approach that includes the smallest phytoplankton groups in its surveys in the North Sea is the FerryBox project implemented by the GKSS (www.ferrybox.org). A FerryBox is an autonomous in situ measurement device that can be operated on board ships of opportunity. It is capable of generating information on oceanographic parameter [9]. In the FerryBox, phytoplankton is characterized on the basis of pigment composition via fluorescence. All phytoplankton groups and size fractions are included in this analysis. However, this approach is only suited for the identification of larger taxonomic algal groups. For instance diatoms and dinoflagellates cannot be distinguished via this approach. However, despite the fact that the FerryBox system lacks information on species composition of the phytoplankton it is a powerfull technology to provide information on phytoplankton occurance with high spatio temporal resolution in the marine environment.

Here we present a project that aims at the combination of molecular sensors with the FerryBox System in order to provide a powerful methodology for species specific in situ surveillance of phytoplankton.

\section{MATERIAL AND METHODS}

\section{Nucleic Acid Biosensor}

Species specific probes for the use in combination with the nucleic acid biosensor have been designed as described previously [10]. The probes were designed using the ARB software package[11] (http://www.arb-home.de). The probes have a length within a range between 18 to 23 bases, with at least two mismatches against all non-target organisms. The ARB database contained over 3000 published and unpublished algal sequences. The hybridisation experiments related to the identification of microalgae with the nucleic acid biosensor have been carried out according to a protocol described previously [12].

\section{Phylochips}

The DNA-microarray (PHYLOCHIP) for the analysis of FerryBox-samples contained a hierachically organized probe set for the identification of major groups within the phytoplankton. The probe design and the hybridisation protocol has been carried out as previously described for analysing the temporal dynamics of prasinophytes at the island of Helgoland $[13] .$.

\section{The FerryBox-System and Sampling}

Oceanographic data in the area of the Southern North Sea have been generated making use of the FerryBox-System developed by the GKSS Research Center in Geesthacht. A FerryBox is an autonomous device located on ships of opportunity that has the capability to autonomously generate information on the plankton composition and a number of other 
parameters for the North Sea [9]. In our project we took advantage of a FerryBox, which is operated on board of the TorDania, a bulk carrier, which is travelling on a regular route between Cuxhaven in Germany and Immingham in the UK.

Water samples have been taken in the Southern Norths Sea at locations with high chlorophyll content identified previously with the FerryBox. Phytoplankton in the water samples was harvested by a filtration onto a filter with a pore size of $0.2 \mu \mathrm{m}$ and subjected to a PHYLOCHIP analysis.

\section{RESULTS}

\section{Nucleic Acid Biosensor}

In the EU-project ALGADEC a portable semi-automated nucleic acid biosensor has been developed, in order to facilitate the surveillance of marine microalgae (www.algadec.net). Originally, the development of the biosensor was dedicated to the detection of toxic algae, but currently we are working on adapting the biosensor to the assessment of phytoplankton key species in the North Sea. With the help of this new technology, based on electrochemical measurements, it is possible to detect microalgae from watersamples in less than two hours, without the need of expensive equipment. The detection of the target species is based on a sandwich hybridisation (Fig. 1) that is carried out on a sensorchip. A sandwich hybridisation is a molecular probe-based method for rapid target identification that uses two molecular probes targeting ribosomal RNA (rRNA). A capture probe bound to a solid surface immobilizes the target ribosomal RNA, which in turn forms a hybrid complex with a second signal probe. Furthermore, an antibodyenzyme complex binds to the signal moiety of the signal probe and reacts with a substrate forming an electrochemical current [14].

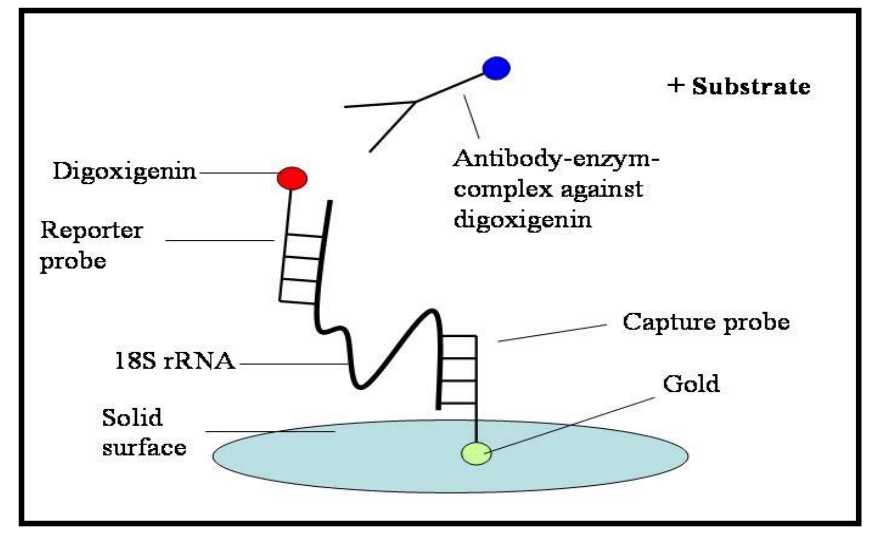

Figure 1. Schematic drawing of a sandwich hybridisation.

The portable nucleic acid biosensor is a semiautomated measurmentdevice, which has approximately briefcase format (Fig. 2). The main steps of the probe to target hybridisation and the analysis process are executed automatically in the sensor device. However, sampling and sample preparation has to be carried out manually. This involves a manual filtration step and the resuspension of the filtered plankton cells in lysis buffer. At the heart of the nucleic acid biosensor there is a multiprobe chip that can be used for the simultaneous detection of 14 different targets plus two controls and thus for the detection of species compositions in water samples.

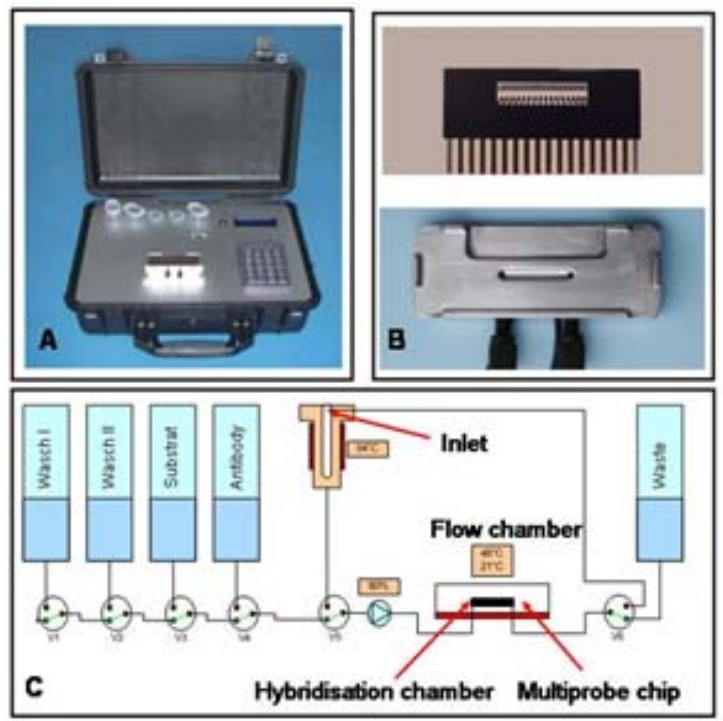

Figure 2: A: Portable biosensor in briefcase format. B: Multiprobe-chip and hybridisation chamber. C: Process chart of the biosensor.

The applicability of the biosensor for the detection of microalgae has been evaluated with laboratory cultures and in the course of an end user workshop. During that workshop contaminated field samples from the Orkney Islands (UK) were sucessfully screened by the end users for the presensce of cells from the genus Pseudo-nitzschia, subsequent to an introduction to the handling manual.

The ALGADEC nucleic acid biosensor represents a device that allows for an automated, inexpensive, easy and rapid detection of marine phytoplankton, based on the binding of ribosomal RNA by species specific molecular probes. The use of ribosomal RNA for the identification of microbes is advantageous becuase the molecule is present in high numbers in a cell, which circumvents an amplification of the target molecule prior to the molecular analysis. Furthermore, the intensity of the electrochemical signal is proportional to the amount of ribosomal RNA bound to the sensor surface. This provides the opportunity for a quantitative analysis. 

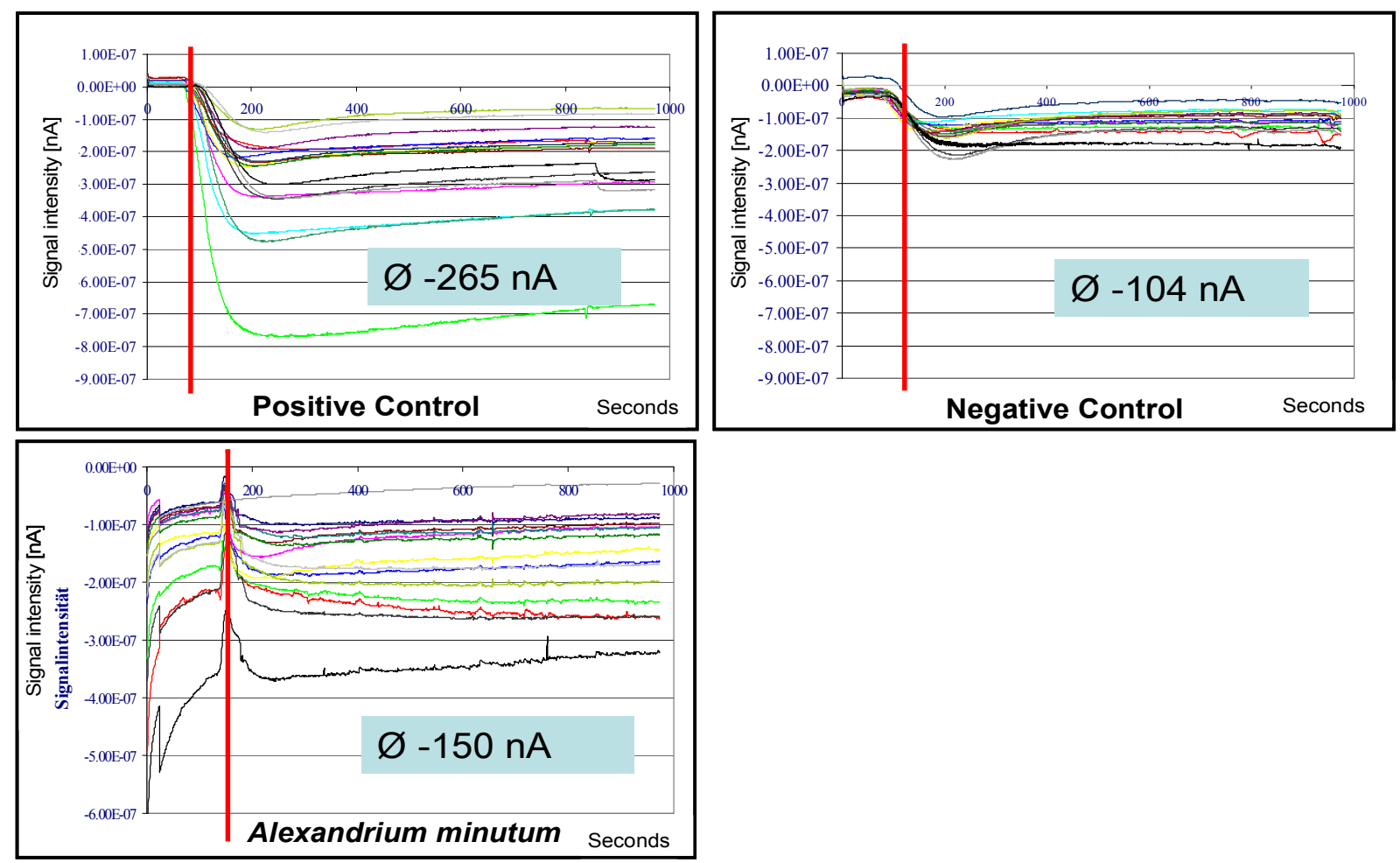

Figure 3: Identification of laboratory cultures of the toxic dinoflagellate Alexandrium minutum with the nucleic acid biosensor.

2. Phylochip Analysis of FerryBox Samples

A PHYLOCHIP analysis of samples taken with a FerryBox system has been carried out in order to assess the diversity within different phytoplankton patches that occur at one day in the area of the southern North Sea. The FerryBox system is installed on the cargo vessel Tor Dania, which is travelling in on a regular route between Cuxhaven in Germany and Immingham in the UK patches of high photosynthetic biomass have been identified via fluorescence measurements that had been carried out by the FerryBox system prior sampling [9]. Here we present an analysis of four different samples that have been taken on May 7, 2008. The PHYLOCHIP contained a hierachically organized probe set for the identification of major phytoplankton groups, e.g. chlorophytes or dinoflagellates at class level. Furthermore some groups like cryptophytes and prasinophytes can be distinguished at lower taxonomic levels e.g. genus level [13]. For the latter taxa a phylochip analysis, which is independent of morphological marker or the size of the taxa, is from particular interest. They represent ecologically important phytoplankton groups which are particularly difficult to adress with conventional methods like light microscopy $[13 ; 15]$. Overall, the PHYLOCHIP analysis revealed that signal/noise ratios $(\mathrm{S} / \mathrm{N})$ of all four samples were in a com- parable range. Furthermore, the compostition of the different samples in respect to the presence of the major groups within the phytoplankton like Chlorophyceae (Chlo02), Cryptophycea (CryptoB), Dinophyceae (DinoE-12) or Prasinophyceae (Pras04) was similar as positive signals could be observed for these groups in all four samples (Fig. 4). However, at lower taxonomic levels for some groups differences in the compostion could be observed. The PHYLOCHIP contained a probe set for the identification of Cryptophycea at class level and to distinguish seven diffent clades in this class [15]. On May 5, 2008 the signal for clade three (Crypt 3-26) was below the $\mathrm{S} / \mathrm{N}$ - threshold of two in sample six (Guillardia sp., Hanusia sp.) and the signal for clade five Crypt 53-25; Proteomonas sp.) was below the threshold in the samples four and six. In contrast, the signal for the probe targeting clade 1 (Cryptomonas) was below the threshold in all four samples. This observation indicates, that this genus does not contribute significantly to the phytoplankton population at May 5, 2008. However, all probes for the other clades displayed signals obove the threshold in all four samples. This indicates that the majority of the different genera (Rhinomonas, Rhodomonas, Storeatula, Plagioselmis, Teleaulax, Geminigera, Komma, Chroomonas, Hemiselmis, Plagiomonas and Falcomonas 

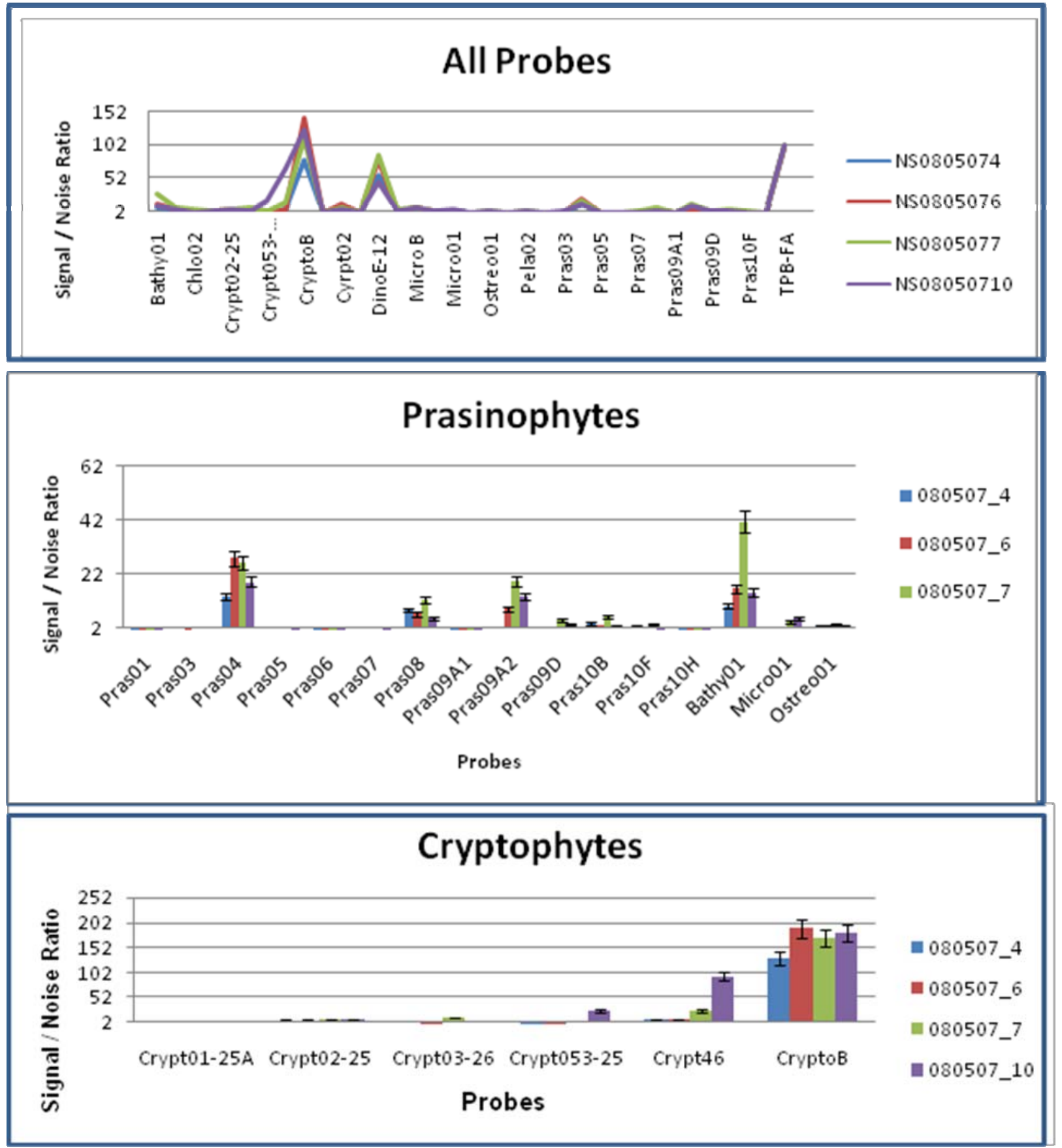

Figure 4: Results of the PHYLOCHIP analysis of the FerryBox-samples from May 05, 2008. The samples were taken at locations with the following coordinates: 080507_4: $53^{\circ} 96.25^{\prime} \mathrm{N}, 03^{\circ} 26.02^{\prime} \mathrm{E}$; 080507_6: $54^{\circ} 03.06^{\prime} \mathrm{N}, 04^{\circ} 28.17^{\prime} \mathrm{E}$; $080507{ }^{2}$ : $54^{\circ} 12.27^{\prime} \mathrm{N}, 05^{\circ} 48.74 \mathrm{E}$; 080507_10: $54^{\circ} 20.81^{\prime} \mathrm{N}, 07^{\circ} 46.28^{\prime} \mathrm{E}$.

could be present in all four samples. However, this is a very broad view and does not give much information on the diversity within the Cryptophytes. Thus it would be desirable if the PHYLOCHIP could be extended by probes that possess the possibility to identify Cryptophytes below the level of clade. This was the case in the probe set for the prasinophyta. The results for the Prasinophyceae indicate that species from the family Mamelliales (Pras04) are present in all four samples. The Mamlliales comprise the genera Bathycoccus (Bathy01), Micromonas (Micro01) and Ostreococcus (Ostreo01). Out of these three genera only Bathycoccus displays a signal above the threshold of two in all four samples, the probe targeting the genus Micromonas displays a clear positive signal in two out of the four samples. However, the probe for Ostreococcus is only 
marginal above the threshold in all four samples. These results indicate that Bathycoccus is the dominating genus among the Mamelliales in the Southern North Sea. This is in accordance with previous observations at the island of Helgoland for the years 2004-2006 [13].

The data that we present here are the results from four experiments that give information about the occurance of the major phytoplankton groups and a number of clades and genera that are assigned to these groups. The generation of the data set required about half a working day, which is significantly less than the amount of time required for a comparable analysis based on conventional methods. Thus the data set demonstrates the power of the PHYLOCHIP approach to facilitate assessment of phytoplankton, including those taxa and size classes e.g. cryptophyta or picoplankter, which have been omitted in ecosystem studies so far.

\section{CONCLUSIONS AND OUTLINE}

The nucleic acid biosensor and the PHYLOCHIP that we present in this publication have great potential for a high throughput assessment of marine phytoplankton. Furthermore, with the FerryBox-system the GKSS carries out already today in situ measurements of oceanographic data in the North Sea.
Hence, a combination of the FerryBox system with the nucleic acid biosensor would enable high resolution monitoring of phytoplankton, complementing the in situ measurements of oceanographic parameters. Thus, we are currently working on implementing this combination of molecular sensors and the FerryBox-system. However, our current data indicate only marginal differences in the composition of different phytoplankton patches, if the assessment is focused on higher taxonomic levels. Therefore, in the future phytoplankton assessment has to be carried out at lower taxonomic levels like genus or species-level. Therefore, we aim at the extension of our probe set for the nucleic acid biosensor and the Phylochip by species specific molecular probes that target key species within the phytoplankton of the North Sea.

\section{ACKNOWLEDGMENT}

The authors would like to thank all partners from the EUProject ALGADEC for excellent cooperation. Sonja Diercks was supported by the EU-project ALGADEC (COOP-CT2004-508435-ALGADEC) of the 6th Framework Program of the European Union and the Alfred Wegener Institute for Polar and Marine Research.

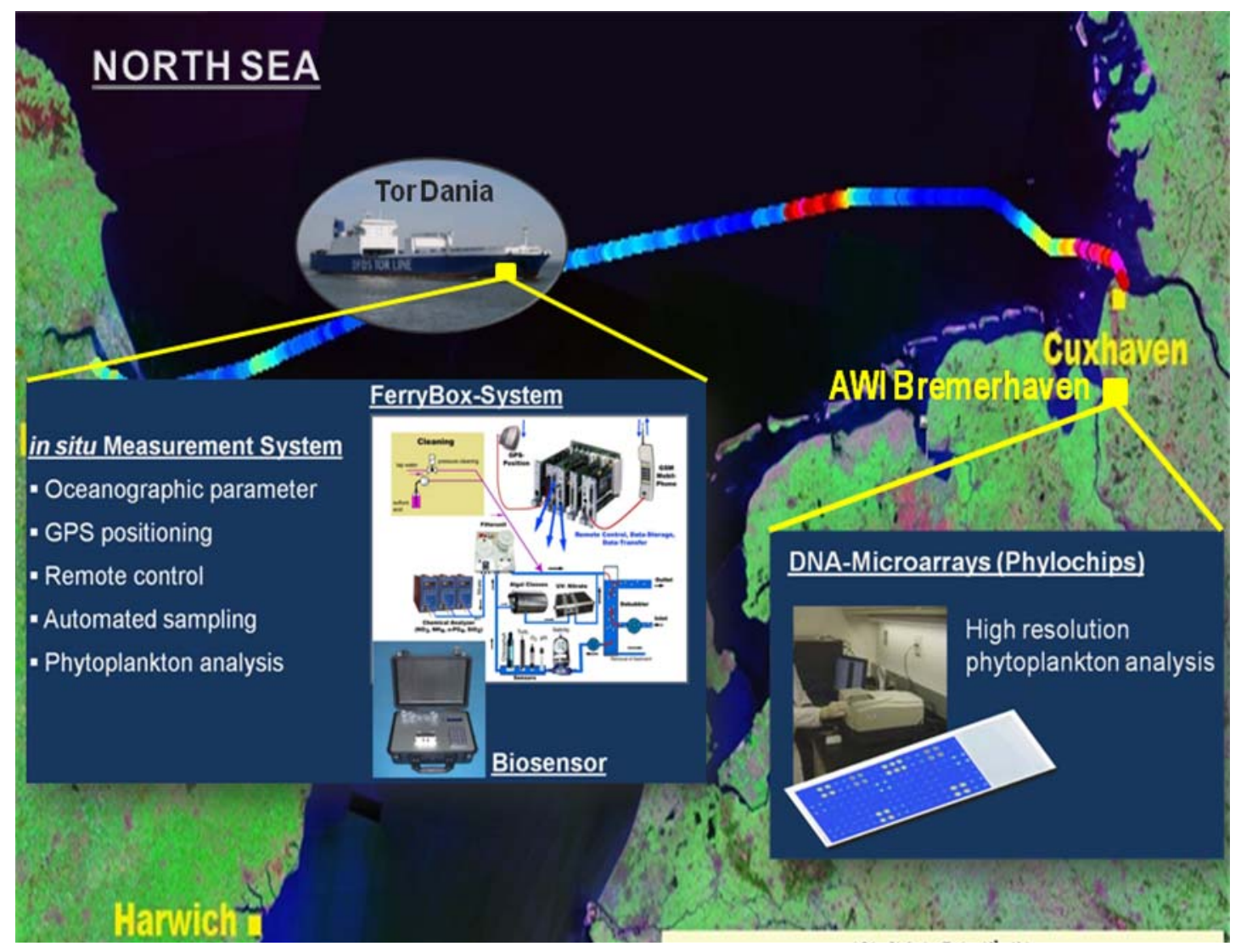

Figure 5: Outline of the approach to combine molecular sensors with the FerryBox-system 


\section{REFERENCES}

[1] M. Edwards, and A.J. Richardson (2004) Impact of climate change on marine pelagic phenology and trophic mismatch, Nature vol. 430, pp. 881-884, 2004.

[2] M.Edwards, G. Beaugrand, P.C. Reid, A.A. Rowden, and M.B. Joes Ocean climate anomalies and the ecology of the North Sea, Mar Ecol Progr Ser vol. 239, pp.1-10, 2004.

[3] R.I. Amann, B.J. Binder, R.J. Olson, S.W. Chisholm, R. Devereux, and D.A. Stahl, Combination of 16S rRNA-targeted oligonucleotide probes with flow cytometry for analyzing mixed microbial populations. Appl Environ Microbiol.vol. 56, pp. 1919-1925, 1990.

[4] C.R. Woese C.R. Bacterial evolution. Microbiological Reviews. vol. 51: pp. 221-271, 1987

[5] S.J. Giovannoni, T.B. Britschgi, C.L. Moyer and K.G. Field, Genetic diversity in Sargasso Sea bacterioplankton. Nature vol. 345, pp. 60-63, 1990.

[6] B.L. Maidak, J.R. Cole, T.G. Lilburn, C.T.J. Parker, P.R. Saxman, R.J Farris, G.M. Garrity, G.J. Olson, T.M. Schmidt, and J.M. Tiedje The RDP-II (Ribosomal Database Project). Nucleic. Acids. Res. vol. 29, pp.173-174, 2001.

[7] R. Groben, U. John, G. Eller, M. Lange, and L.K. Medlin Using fluorescently- labelled rRNA probes for hierarchical estimation of phytoplankton diversity. Nova Hedwigia. vol. 79, pp. 313-320, 2004.

[8] K.Metfies, K., and L.K. Medlin, L.(2004). DNA Microchips for Phytoplankton: The Flurorescent Wave of the Future, Nova Hedwigia, vol. 79, pp. 321-327, 2004.

[9] W. Petersen, H. Wehde, H. Krasernann, F. Colijn, and F. Schroeder (2008). FerryBox and MERIS - Assessment of coastal and shelf sea ecosystems by combining in situ and remotely sensed data. ESTUARINE COASTAL AND SHELF SCIENCE vol. 77: pp. 296-307, 2008.

[10] S. Diercks, S., L.K. Medlin, and K. Metfies, Colorimetric detection of the toxic dinoflagellate Alexandrium minutum using sandwich hybridization in a microtiter plate assay, Harmful algae vol. 7, pp. 135$147,2008$.

[11] W. Ludwig, O. Strunk, R. Westram, L. Richter, H. Meier, V. Yadhukumar, A. Buchner, T. Lai, S. Steppi, G. Jobb, W. Förster, I Brettske, S. Gerber, A.W. Ginhart, O. Gross, S. Grumann, S. Hermann, R. Jost. A. König, T. Liss, R. Lüßmann, M. May, B. Nonhoff, B. Reichel, R. Strehlow, A. Stamatakis, N. Stuckmann, A. Vilbig, M. Lenke, T. Ludwig, A. Bode, and K.H. Schleifer ARB: A Software Environment for sequence data. Nucleic Acids Res. Vol. 32, pp. 1363 1371,2004

[12] S. Diercks, K. Metfies, and L.K. Medlin Development and adaptation of a multiprobe biosensor for the use in a semi-automated device for the detection of toxic algae. Biosensors \& Bioelectronics vol. 23, pp. 1527$1533,2008$.

[13]C. Gescher, K. Metfies, S. Frickenhaus, B. Knefelkamp, K.H. Wiltshire, and L.K. Medlin Assessing Community Composition of Prasinophytes at the Helgoland Reede Sampling Site with a DNA Microarray: A Feasibility Study. Appl. Env. Microb. Vol 74, pp. 5305-5316, 2008

[14]K. Metfies, S. Huljic, M. Lange, and L.K. Medlin Electrochemical detection of the toxic dinoflagellate Alexandrium ostenfeldii with a DNA-biosensor, Biosensors \& Bioelectronics vol. 20, pp.1349-1357, 2005.

[15]K. Metfies, K., and L.K. Medlin, L.K. Refining Cryptophyte Identification with DNA-Microarrays, Journal of Plankton Research vol.29, pp. 1071-1075, 2007 